\title{
El interés de la justicia en el Estatuto de Roma: un análisis a partir de las decisiones en la situación de Afganistán
}

The Interests of Justice in the Rome Statute: An Analysis from the Decisions in the Situation of Afghanistan

0 interesse da justiça no Estatuto de Roma: uma análise a partir das decisões sobre a situação no Afeganistão

Alejandro Sánchez Frías*

Fecha de recepción: 19 de abril de 2021

Fecha de aprobación: 22 de julio de 2021

Doi: https://doi.org/10.12804/revistas.urosario.edu.co/anidip/a.10499

Para citar este artículo: Sánchez Frías, A. (2022). El interés de la justicia en el Estatuto de Roma: un análisis a partir de las decisiones en la situación de Afģanistán. ANIDIP, 9, 1-28. Doi: https://doi.oro̧/10.12804/ revistas.urosario.edu.co/anidip/a.10499

\section{Resumen}

El primer acercamiento jurisprudencial en las dos décadas de historia de la Corte Penal Internacional (CPI) al requisito del "interés de la justicia" ha generado fuertes críticas de la doctrina y la sociedad civil. Frente a la interpretación restrictiva del concepto de "interés de la justicia", defendida de manera abstracta por un importante sector doctrinal, la Sala de Cuestiones Preliminares en la situación de Afganistán dio sentido a este concepto a través de una polémica interpretación extensiva, la cual no ha sido rechazada de forma explícita por la Sala de Apelaciones. El presente

\footnotetext{
Profesor en el área de derecho internacional público y relaciones internacionales de la Universidad de Málaģa. Doctor en derecho y máster en aboģacía por la Universidad de Málagaa, máster en estudios europeos por la Universidad de Salamanca y máster en derecho europeo por el Coleģio de Europa (Brujas). El presente artículo se enmarca en el proyecto de investiģación Regímenes de facto, desterritorialización de las obliǵaciones de los Estados y consecuencias para la seguridad, dignidad humana y sostenibilidad del Ministerio de Economía y Competitividad, y en el grupo de investigación andaluz Protección Internacional de los Derechos Humanos, Seguridad y Medio Ambiente, ambos diriģidos por la Dra. Ana Salinas de Frías. El autor quisiera mostrar su aģradecimiento al jurado del X Certamen de Ensayos Blattmann, Odio Benito y Steiner por haberle otorgado el tercer puesto a este trabajo, así como a los tres revisores anónimos del ANIDIP por sus suģerencias y comentarios.
} 
trabajo analiza los elementos jurídicos y económicos presentes en esta interpretación expansiva para determinar las luces y sombras que se pueden causar a la hora de tomar una decisión de tal calado como la de determinar qué constituye el "interés de la justicia".

Palabras clave: Corte Penal Internacional; apertura de la investigación proprio motu; interés de la justicia; interpretación restringida; interpretación amplia.

\begin{abstract}
The first jurisprudential approach to the requirement of the "interests of justice" in the two decades of history of the International Criminal Court (ICC) has caused strong criticism among the scholarship and the civil society. While an important sector of academia has defended a restrictive approach to the interpretation of the "interests of justice", the Pre-Trial Chamber decided, in the situation of Afghanistan, to give sense to this concept in an expansive manner, an approach not expressly disregarded by the Appeals Chamber. The present study analyses the legal and economic elements used in this expansive interpretation to determine the lights and shadows of using that approach in the definition of an element as important as the "interests of justice".
\end{abstract}

Keywords: International Criminal Court; opening of an investigation proprio motu; interests of justice; restrictive interpretation; expansive interpretation.

\title{
Resumo
}

A primeira abordagem jurisprudencial nas duas décadas de existência do Tribunal Penal Internacional à exigência do "interesse da justiça" tem gerado fortes críticas por parte da doutrina e da sociedade civil. Diante da interpretação restritiva do conceito de "interesse da justiça" defendido de forma abstrata por um importante setor doutrinário, a Câmara de Pré-Julgamento na situação do Afeganistão deu sentido a esse conceito, por meio de uma ampla interpretação polêmica, a qual não foi explicitamente rejeitada pela Câmara de Recursos. Este trabalho analisa os elementos jurídicos e econômicos presentes nesta interpretação expansiva para determinar as luzes e sombras que podem causar no momento de tomar uma decisão de tal profundidade, a que determina o que constitui o "interesse da justiça".

Palavras-chave: Tribunal Penal Internacional; abertura de inquérito proprio motu; interesse da justiça; interpretação restrita; interpretação ampla. 


\section{Introducción}

Con 123 Estados parte, el Estatuto de Roma (ER) marca en su preámbulo un objetivo tan ambicioso como necesario: "que los crímenes más graves de trascendencia para la comunidad internacional en su conjunto no deben quedar sin castigo y que, a tal fin, hay que adoptar medidas en el plano nacional e intensificar la cooperación internacional para asegurar que sean efectivamente sometidos a la acción de la justicia”. Justo después de cumplir veinte años de su adopción, la solicitud de apertura de investigación en el asunto de Afganistán puso duramente a prueba la capacidad de actuación de la Corte Penal Internacional (CPI). Por un lado, Estados Unidos amenazó y cumplió con su amenaza de ejecutar medidas contra funcionarios de la CPI por la investigación de actos cometidos por personal estadounidense ("United States Imposes”, 2021). A esta prueba de capacidad se sumó otra de identidad que, como veremos, surgió inesperadamente en el seno de este procedimiento: la del concepto de "interés de la justicia” en el marco del artículo 53. 1. c del ER.

En su obra, ¿Qué es justicia?, Kelsen (1991) concluyó, que

[n]inguna otra cuestión se ha debatido tan apasionadamente, ninguna otra cuestión ha hecho derramar tanta sangre y tantas lágrimas, ninguna otra cuestión ha sido objeto de tanta reflexión para los pensadores más ilustres, de Platón a Kant. Y, sin embargo, la pregunta sigue sin respuesta" (p. 35).

Partiendo de esta conclusión, el jurista y filósofo austríaco acepta que no puede ofrecer una definición absoluta de este concepto, sino una basada en los valores particulares de quien propone la definición: "verdaderamente, no sé ni puedo afirmar qué es la Justicia, la Justicia absoluta que la humanidad ansía alcanzar. Solo puedo estar de acuerdo en que existe una Justicia relativa y puedo afirmar qué es la Justicia para mí" (Kelsen, 1991, p. 63).

Del mismo modo, los órganos de la CPI tampoco pueden ofrecer una respuesta definitiva ni absoluta sobre qué es la justicia ni cuáles son sus intereses a la hora de fijar el concepto: interés de la justicia. Solo puede presentar lo que, conforme a los principios recogidos por el ER, debe significar la justicia para la misión de la CPI y, en concreto, como requisito para la apertura de una investigación a efectos del artículo 53 del ER. Dado que este tratado no define el "interés de la justicia”, y no existía ningún pronunciamiento judicial hasta los que ocupan el objeto de este estudio en $2019 \mathrm{y}$ 2020, la doctrina ha tenido la oportunidad de ofrecer múltiples interpretaciones que hasta la fecha no habían sido confirmadas ni rechazadas de manera taxativa por la 
jurisprudencia. En un trabajo publicado poco después de la entrada en vigor de este instrumento fundacional, Gómez Colomer (2002) ya anunciaba:

es difícil explicar qué debe entenderse por "redundar en interés de la justicia”. Esta frase, de origen anglosajón y a la que el EstCPI se refiere varias veces en relación a actos o momentos distintos, no es nada clara, y va a ser muy importante lo que diga la CPI al respecto en el futuro, puesto que en realidad es una norma procesal que otorga una facultad discrecional a su destinatario (p. 300).

El presente estudio tiene como objetivo determinar qué elementos conforman el interés de la justicia a efectos de la apertura de una investigación bajo el artículo 53. 1. c del ER desde la óptica de la nueva jurisprudencia derivada del asunto de Afganistán. Tras introducir brevemente en la sección primera las corrientes doctrinales existentes y los pronunciamientos de la Sala de Cuestiones Preliminares (sCP) y de la Sala de Apelaciones (sA) a este respecto, analizaremos cada uno de los elementos específicos empleados por cada una de estas, destacando sus debilidades y fortalezas desde un punto de vista jurídico y, sobre todo, desde la perspectiva del derecho internacional. Para ello, la sección segunda, la más extensa dadas las polémicas novedades que se introducen en el debate sobre el "interés de la justicia”, se centrará en los elementos temporales, económicos y de cooperación propuestos por la SCP para rechazar la existencia del interés de la justicia. La sección tercera, por su parte, se dedica al análisis de los elementos requeridos por la sA - la gravedad de los crímenes y los intereses de las víctimas-, destacando además cómo esta decisión ha zanjado la discusión sobre si estos dos elementos son definitorios del interés de la justicia o si, por el contrario, sirven como contrapeso. A la vista del análisis crítico, ofreceremos nuestras conclusiones sobre cuáles elementos deben ser introducidos y cuáles no a la hora de definir el interés de la justicia en futuros procedimientos.

\section{El debate doctrinal previo y las dos}

\section{posturas en el asunto de Afģanistán}

Antes de que surgiese el debate en torno al asunto de Afganistán, De Souza Días (2017) identificó dos corrientes principales según los elementos que pueden incluirse en el concepto del "interés de la justicia” (pp. 732-733). La primera de ellas defiende una interpretación restringida de dicho concepto, según la cual la fiscalía podría rechazar una investigación por este motivo únicamente cuando concurran los elementos expresamente mencionados en el artículo 53 del ER: la gravedad de los crímenes, los intereses de las víctimas, la edad y salud del acusado y el papel 
asumido en la comisión de los presuntos crímenes. La segunda corriente se inclina por una interpretación más amplia del criterio que permitiría a la fiscalía rechazar la apertura de una investigación por las razones indicadas en el ER y por elementos no relacionados estrictamente con el procedimiento penal en curso. Esto incluiría, como veremos, elementos temporales, el uso eficiente de los escasos recursos económicos y personales que tiene la CPI a su disposición y el nivel de cooperación de los Estados en la persecución de los crímenes en cuestión.

No ha sido sino hasta la llegada de las dos decisiones que formarán el objeto de nuestro estudio que la aplicación de estas corrientes se ha visto puesta a prueba. La decisión del 12 de abril de 2019 en el asunto de Afganistán recoge el primer análisis jurisprudencial detallado del requisito del "interés de la justicia” para la apertura de una investigación conforme al 53. 1. c del ER (Sala de Cuestiones Preliminares II, 2019). De las cuatro páginas que dedica a este punto se pueden extraer los tres elementos que respaldan la negativa a autorizar la investigación del asunto de Afganistán “en interés de la justicia”: el lapso transcurrido entre el inicio de estos crímenes (2005) y la fecha en la que se solicita la apertura de la investigación (Sala de Cuestiones Preliminares II, 2019 par. 93); la falta de cooperación de los Estados implicados, que indica que una investigación exitosa será poco factible (Sala de Cuestiones Preliminares II, 2019, par. 94), y que la dedicación de los limitados recursos de los que dispone la CPI a este asunto perjudicaría la apertura de otras investigaciones más realistas o factibles (Sala de Cuestiones Preliminares II, 2019, par. 95).

Este pronunciamiento, considerado por algunos como "el anticlímax tras más de una década de examen preliminar por la Oficina del Fiscal, y un año y medio de deliberaciones judiciales"' (Vasilev, 2019), fue recurrido por la fiscalía debido, entre otras razones, al polémico análisis del interés de la justicia llevado a cabo por la SCP. En su decisión, la SA determinó que la SCP se había extralimitado en sus funciones a la hora de examinar el "interés de la justicia” y que, por lo tanto, no era necesario realizar un análisis de fondo. ${ }^{2}$ No obstante, si bien en un obiter dictum motivado por el fuerte debate que produjo en la academia y la sociedad civil la decisión impugnada (Sala de Apelaciones, 2020, par. 48), esta sala señaló: 
The Pre-Trial Chamber's reasoning in support of its conclusion regarding the 'interests of justice' was cursory, speculative and did not refer to information capable of supporting it. [...] There is no indication that the Pre-Trial Chamber considered the gravity of the crimes and the interests of victims as articulated by the victims themselves in conducting this assessment. In these circumstances, the Appeals Chamber is of the view that the Pre-Trial Chamber did not properly assess the interests of justice (Sala de Apelaciones, 2020, par. 49).

En este solitario párrafo, la sa se limitó a indicar que en el "interés de la justicia" deben tenerse en cuenta los intereses de las víctimas y la gravedad del crimen, alineándose así, como veremos, con quienes abogan por una interpretación restrictiva de este concepto. No obstante, y pese a que una lectura rápida pudiese dar la impresión de que se rechazan los elementos utilizados por la SCP, una segunda lectura muestra que no es el caso. La SA considera que la SCP fue especulativa al no contar con suficiente información para confirmar su análisis, pero en ningún momento estableció que, en abstracto, la inclusión de los tres elementos mencionados fuese errónea.

La respuesta de la SA, en su brevedad, no cierra la puerta a interpretaciones más amplias del interés de la justica, cuestión importante de destacar por dos razones de peso. En primer lugar, porque deja abierta la posibilidad de que, cuando la SCP examine casos en los que la fiscalía decida no abrir una investigación "en interés de la justicia", la SCP pueda revisar la presencia de elementos como la extensión de la etapa preliminar, la cooperación de los Estados o el uso eficiente de los recursos de la CPI. En segundo lugar, porque podría ser empleada por la propia fiscalía no solo en investigaciones propio motu, sino también para rechazar las referidas por el Consejo de Seguridad de la Organización de Naciones Unidas - CSNU(Dannenbaum, 2020, p. 151). Los próximos apartados se dedicarán por tanto a analizar, desde distintos puntos de vista, cada uno de estos elementos y el papel que pueden jugar en las futuras líneas de actuación de la CPI, comenzando por los explícitamente señalados por la SCP y pasando posteriormente a los de la SA.

\section{La interpretación extensiva de la SCP}

A la hora de decidir que una autorización en Afganistán no redundaría en el interés de la justicia, la SCP tuvo en cuenta, como hemos visto, tres elementos principales: el tiempo transcurrido entre la presunta comisión de los crímenes y la solicitud de inicio de la investigación; la falta de cooperación de los Estados implicados, y el riesgo de perder recursos que la CPI podría utilizar en la investigación de asuntos 
más realistas o factibles. Ninguno de estos elementos es mencionado en el artículo 53 del ER y todos ellos escapan, además, a lo que consideraríamos la vertiente estrictamente retributiva de la justicia penal.

Como puede imaginarse, la decisión de la scP de elegir estos tres elementos no surge de la nada, sino que se inspira en ciertas corrientes que defienden una aproximación expansiva de los elementos que deben conformar la justicia penal internacional. En las próximas secciones analizaremos las fortalezas y debilidades de cada uno de estos elementos según los defienden algunos participantes en el proceso ante la SA de la CPI, que formarían un concepto del interés de la justicia que incluye “consideraciones más amplias que la simple justicia criminal” (CPI, 2019, p. 93).

\subsection{El lapso entre la comisión del crimen y la solicitud de apertura de la investigación}

El primer elemento tomado en consideración por la SCP es el lapso transcurrido entre la presunta comisión de los crímenes en Afganistán y la solicitud de inicio de la investigación por parte de la fiscalía de la CPI. Como recordaremos, la fiscalía inició el examen preliminar en el año 2006, pero solo hasta 2017 solicitó la investigación de los crímenes presuntamente cometidos en Afganistán a partir del año 2003 y en otros Estados parte a partir del año 2002.

Tal y como indica la SCP en su decisión, la duración de la fase de examen preliminar, por un total de 11 años, supone un periodo particularmente largo (Sala de Cuestiones Preliminares, 2017, par. 92). La scP considera que el transcurso de este tiempo pone seriamente en duda el posible éxito de la investigación y, con ello, aporta el primer elemento del "interés de la justicia” que no está presente en el asunto de Afganistán. La pretensión de intentar llevar a buen puerto una investigación catorce años después de la comisión de los crímenes sería un ejemplo claro de la aplicación del aforismo americano "justice delayed, justice denied" - justicia retrasada, justicia negada- (United States c. Hastings, 1998) a la CPI. Para la SCP esta es la primera señal de que no es conforme al "interés de la justicia” proceder con tal retraso.

Las dilaciones en la etapa preliminar se encuentran presentes de forma habitual en los asuntos más complejos de los que conoce la fiscalía de la CPI (Magnoux, 2017; Bosco, 2020; Olásolo Alonso, 2012). Véase, por ejemplo, el asunto de la República Centroafricana en el que, tras dos años sin recibir noticias sobre los resultados de la fase preliminar, la SCP se vio obligada a señalar a la fiscalía que "the preliminary examination of a situation [...] must be completed within a reasonable time [...], regardless of its complexity" (Sala de Apelaciones, 2006, p. 4). Cabe recordar que, 
aunque el ER no impone a la fiscalía un tiempo límite para el desarrollo de la fase preliminar, el propósito de esta etapa y los derechos de las partes aconsejan que se complete en un periodo de tiempo razonable (Olásolo Alonso, 2005, p. 144). La propia fiscalía reconoce que las posibilidades de obtener y asegurar las pruebas con éxito son mayores mientras menor sea el tiempo de llegada a la escena del crimen:

The time gap between events on the ground and the moment when the Office can investigate can result in loss of evidence. This is contrary to the "golden hour" principle which recognises that the sooner one can be present at the crime scene, the higher the chances are that better quality evidence and leads will be discovered and secured (Oficinal del Fiscal, 2015, par. 24).

Los retrasos en este proceso pueden resultar bien de la propia naturaleza del asunto, bien de una estrategia de complementariedad positiva con la que la fiscalía pretende presionar a los Estados parte para que conduzcan investigaciones domésticas de forma eficaz (Dancy \& Montal, 2017). En cualquier caso, lo cierto es que parece ser que la duración de la fase preliminar depende en gran medida del actor que ha iniciado el proceso: tres semanas y media en el caso de asuntos referidos por el CSNU; entre cuatro y diez meses para los presentados por los Estados parte, y a partir de 5 años para los asuntos iniciados motu proprio por la fiscalía, lo cual suele ser resultado de la falta de cooperación del Estado parte implicado en el asunto y que no lo ha referido por sí mismo (Pues, 2017, pp. 438-439).

Pese a la existencia de precedentes con una dilatada etapa preliminar, el asunto de Afganistán es el primero en el que la SCP rechaza la apertura de una investigación basándose en este criterio temporal. ¿Podemos decir que su inclusión en el “interés de la justicia" está suficientemente motivada? La representación legal de las víctimas critica precisamente que la SCP no basara su decisión ni en el ER ni en los trabajos preparatorios ni en la jurisprudencia previa, resaltando además:

To conclude that too much time has passed since the period 2005-2015 was unreasonable and an abuse of discretion. The successful prosecutions of Khmer Rouge leaders by the ECCC, with trials starting in 2009, for crimes committed in 1975-1979, demonstrate that probative evidence and suspects can remain available for decades. World War II-era trials have famously taken place in every decade since the war. By reading into the Statute a criterion of 'feasibility' that does not appear in it, the Chamber exceeded its discretion and unfairly deprived the Victims of their only 
chance of investigation and prosecution ("Legal representatives", 2019, par. 55).

La toma en consideración de este criterio a la hora de denegar una investigación manda, desde nuestro punto de vista, un peligroso mensaje sobre la actuación y el futuro de la CPI. Tal y como indican las víctimas, existen precedentes de investigaciones relativamente exitosas de crímenes internacionales cometidos hace más de treinta años. La CPI, por su propia naturaleza basada en el principio de complementariedad, se enfrenta a asuntos de gran complejidad en Estados que no pueden o no quieren llevar a cabo una investigación y enjuiciamiento efectivos y con los que la cooperación puede resultar un obstáculo difícil de salvar en un tiempo razonable. La presencia de la temporalidad en el requisito del "interés de la justicia”, ya sea por la fiscalía o por la SCP, pondría seriamente en peligro otros asuntos que se encuentran en fase preliminar - en ocasiones, debido a la propia estrategia seguida por la fiscalía para propiciar la apertura de procedimientos nacionales (Seils, 2015) - y acrecentaría las críticas contra su sistema de selección de asuntos.

\subsection{La cooperación estatal}

La falta de cooperación de los Estados implicados es el segundo elemento que toma en cuenta la SCP en su decisión. En varios de sus alegatos durante las distintas visitas ante la SA, el representante del European Centre for Law and Justice (ECLJ) defendió la decisión de la SCP a la hora de incluir la falta de cooperación de los Estados implicados como elemento determinante para rechazar el interés de la justicia en la investigación sobre el asunto de Afganistán. Según el ECLJ, así como de acuerdo con el Jerusalem Institute of Justice, el International Legal Forum, My Truth, el Simon Wiesenthal Centre, The Lawfare Project y los uk Lawyers for Israel, ${ }^{3}$ varias razones basadas en normas de derecho internacional y derecho interno respaldarían la decisión de rechazar el interés de la justicia de una investigación que no cuenta con la cooperación de los Estados implicados.

La primera razón por la que esta debería tenerse en cuenta surgiría de la máxima de derecho internacional consuetudinario "pacta tertiis nec nocent nec prosunt". Como bien sabemos, este es un principio básico que cuenta con el apoyo casi universal de los Estados y que ha quedado codificado en el artículo 34 de la Convención de Viene sobre el Derecho de los Tratados (CVDT) en los siguientes términos: "un tratado no crea obligaciones ni derechos para un tercer Estado sin su consentimiento". El ECLJ aplica este principio en dos etapas. En primer lugar, para afirmar que los

3 En adelante, nos referiremos a este grupo con representación común a la cPI únicamente como Jerusalem Institute of Justice. 
Estados que no son partes del ER, como es el caso de Estados Unidos en el presente asunto, no tendrían obligación alguna de cooperar con la CPI (2019, p. 101). En segundo lugar, utiliza este principio para defender que el establecimiento de jurisdicción de la CPI sobre nacionales de Estados que no forman parte del ER (artículo 12. 2. a) va en contra de este principio consuetudinario; llega incluso a calificar este precepto como de "nulo" y ultra vires por crear obligaciones a Estados que han rechazado someterse a la jurisdicción de la CPI. En esta situación, los Estados parte no podrían delegar su jurisdicción a la CPI, dado que habría que esperar a la existencia de un consenso suficiente para que se forme una norma consuetudinaria que permita a los Estados delegar a la CPI su jurisdicción sobre nacionales de Estados no parte (CPI, 2019, p. 101). En una línea similar se posiciona el representante del Jerusalem Institute of Justice:

The interests of justice oblige the Court to be mindful that whereas between its relationship with States Parties [...], the system of cooperation under Part 9 of the Statute is a self-contained regime. [...]

Between the Court and non-States Parties we must identify the specific rules of customary law that apply to that separate circumstance. And the ICC does not operate in a legal vacuum (CPI, 2019, p. 99).

El principio general según el cual los Estados no quedan obligados a cooperar con organizaciones internacionales de las que no son parte no arroja demasiadas sombras (Vierdag, 1982). No obstante, desde el derecho internacional, no podemos coincidir cuando trasladamos el principio general al caso concreto. El ECLJ parece confundir la inexistencia de obligaciones convencionales con respecto a terceros Estados con los efectos que pueden tener los tratados, en este caso el ER, en terceros Estados. Tal y como señalan Dörr y Schmalenbach (2018), las obligaciones convencionales strictu sensu son aquellas que se dirigen directamente a uno o más terceros Estados y le imponen el deber de comportarse de un cierto modo, mientras que los efectos convencionales se refieren al impacto de naturaleza meramente fáctica que puede tener un tratado en los intereses de un tercer Estado (pp. 613-615), siendo únicamente las primeras las que son contrarias al principio de pacta tertiis.

El artículo 12.2. a del ER recoge que la CPI podrá establecer su competencia con respecto a los crímenes a los que se refiere el artículo 5, siempre y cuando sean cometidos en el territorio de un Estado parte. ${ }^{4}$ Esto implica la posibilidad, naturalmente, de que establezca su competencia sobre crímenes cometidos por nacionales

4 Sobre el concepto de "territorio" a estos efectos véase el estudio en detalle de Vaģias (2014). 
de Estados no parte en el territorio de un Estado parte, como es el caso de Estados Unidos y Afganistán, respectivamente. Danilenki (2000) ya señalaba esta posibilidad antes incluso de que entrase en vigor el ER:

Los Estados no miembros no tendrán la posibilidad de bloquear el enjuiciamiento de sus nacionales. Aunque solo los nacionales de los terceros Estados, y no los terceros Estados en sí mismos, serán acusados ante la CPI, es obvio que las actividades de esta nueva institución afectarán a los intereses legales de los Estados no miembros (p. 448). ${ }^{5}$

Tal y como hemos señalado anteriormente, el ER no fuerza a los Estados a cooperar con la CPI, de modo que Estados Unidos no tiene obligación alguna de cooperar en una investigación contra sus nacionales o los nacionales de cualquier otro Estado. ${ }^{6}$ En cambio, sí puede tener un efecto como el que se plantea en el asunto de Afganistán, de forma que se inicie una investigación contra nacionales de Estados no parte que presuntamente hayan cometido crímenes contemplados por el ER en un Estado parte.

En cuanto a la segunda etapa de la argumentación del ECLJ según la cual los Estados parte no pueden delegar su competencia a la CPI en estos casos es, desde el punto de vista del derecho internacional y a nuestro juicio, errónea. No menos importante que el principio pacta tertiis, con un carácter definitivamente consuetudinario, es el principio de la territorialidad. Según este principio, el Estado en cuyo territorio - esto es, tierra, mar y espacio aéreo- ha ocurrido el crimen o en el que se ha cometido uno posee jurisdicción penal sobre este, independientemente de la nacionalidad del sospechoso (Ambos, 2016, p. 211). Nos encontramos ante el principio clásico de la jurisdicción penal por una razón práctica de política criminal: generalmente, el Estado del territorio es el más próximo al crimen y el que está mejor preparado para investigarlo y perseguirlo (Bantekas, 2010, p. 233). Este principio se recoge en sentencias de la Corte Permanente de Justicia Internacional (CPJI) tan conocidas como las del asunto Lotus, en la que puso de relieve que la jurisdicción es ciertamente territorial (CPJI, 1927, p. 18). Incluso en asuntos clásicos de la jurisprudencia estadounidense se establece que la jurisdicción territorial es necesariamente exclusiva y absoluta (Schooner Exchange c. McFaddon, 1812).

Alģunos autores lleģan defienden el deber de cooperación de Estados no parte en ciertas situaciones, como en caso de asuntos referidos por el csnu o que afecten al artículo 1 común de los Convenios de Ginebra. Véase al respecto Wenqi (2006). 
Sin entrar en la clásica discusión acerca de si el ER se basa o no en el principio de jurisdicción universal - lo cual le permitiría operar en ausencia de los vínculos de nacionalidad y territorialidad expresamente mencionados en su artículo 12-, nada parece impedir que los Estados deleguen el ejercicio de la jurisdicción en casos en los que, como el de Afganistán, está presente el principio consuetudinario de territorialidad. Al contrario, no es extraño en derecho internacional que los Estados ejerzan su jurisdicción sobre nacionales extranjeros e incluso que la deleguen a otros Estados sin el consentimiento del Estado de la nacionalidad cuando se cumplen principios establecidos en tratados bilaterales o multilaterales de cooperación penal, incluyendo no solo el de territorialidad, sino también los de nacionalidad de la víctima, la protección de los intereses estatales y la representación (Ryngaert, 2015, pp. 29-41; Bantekas, 2011).

Ahora bien, ¿puede extenderse este poder de delegación a terceros Estados también a tribunales internacionales? Tras analizar los precedentes de otros tribunales penales internacionales, así como de la Comisión Central para la Navegación del Rhin de 1868, Akande (2003) concluye

Los precedentes discutidos anteriormente son prueba de la extensiva práctica de los Estados de delegación de parte de su jurisdicción penal sobre ciudadanos extranjeros bien a otros Estados o a tribunales creados por acuerdos internacionales, en circunstancias en las que no se ha intentado obtener el consentimiento del Estado de la nacionalidad. Esta práctica, junto con la falta de objeciones de los Estados de la nacionalidad de los acusados, señala a una aceptación general de la legalidad de la delegación de la jurisdicción penal (pp. 663-664). ${ }^{7}$

En cualquier caso, la inexistencia de una obligación de cooperar de los Estados no parte y la nulidad de cualquier precepto convencional que implique el inicio de investigaciones contra los nacionales de un Estado no parte es solo el primer argumento del que se sirve el ECLj para afirmar su postura en cuanto a que es contrario al interés de la justicia tratar de imponer la jurisdicción de la CPI sobre los nacionales de Estados que, conforme a las normas consuetudinarias, están en su derecho a no cooperar (CPI, 2019, pp. 102-104).

Otra razón para respaldar la inclusión del grado de cooperación de los Estados en concepto de "interés de la justicia" se centra en los tratados existentes entre Estados Unidos y Afganistán. Paradójicamente, el ECLJ apoya aquí que Afganistán ceda

Traducción propia. 
su jurisdicción, en este caso a Estados Unidos, a través de distintos status of force agreements (SOFA). Según estos acuerdos, firmados entre el gobierno Afganistán y los Estados Unidos en los años 2002, 2003 y 2014, el personal estadounidense "will under all circumstances and at all times be subject to the exclusive jurisdiction of their respective national elements in respect of any criminal or disciplinary offences which may be committed by them on the territory of Afghanistan" (CPI, 2019, pp. 102-104).

Según el ECLJ, la aplicación del principio de lex specialis, recogido en el artículo 30. 4. b de la CVDT, estos tratados bilaterales gobiernan las relaciones entre ambos Estados, por encima de las obligaciones asumidas en el marco del ER (CPI, 2019, pp. 102-104). A ello se suma el argumento de cierto sector doctrinal según el cual la cesión por parte de Afganistán de su jurisdicción sobre crímenes cometidos por nacionales extranjeros al Estado de la nacionalidad implica, por aplicación del principio nemo dat quod non habet, que Afganistán no puede ceder a través del ER algo que ya no posee:

el acto de transferir jurisdicción territorial a un Estado que no es parte del Estatuto de Roma puede realizarse de manera perfectamente acorde con la CVDT si el Estado del territorio tiene una base válida para reclamar la jurisdicción en el momento de comisión de la presunta ofensa. En este sentido, el Estado del territorio transfiere su autoridad de la misma manera que el copropietario de una casa decide vender o transferir su derecho de propiedad sin el consentimiento del otro copropietario (Newton, 2016, p. 399). ${ }^{8}$

No obstante, desde el punto de vista de la fiscalía - así como del de la SCP, este es uno de los pocos puntos en los que coinciden (Sala de Cuestiones Preliminares II, 2019, par. 59) - , el hecho de que exista un sofa con estas características no afecta a la jurisdicción de la CPI, sino que es una prueba más para tener en cuenta en el examen de admisibilidad sobre la incapacidad o falta de voluntad del Estado territorial de perseguir ciertos crímenes cometidos por ciertas categorías de personas o grupos ("Request for leave the appeal", 2019, par. 46). Aquí optamos por respaldar esta aproximación ya que el argumento del ECLJ olvida la vital distinción entre la jurisdicción legislativa - en inglés, jurisdiction to prescribe-, que determina los derechos y límites del Estado para la creación y aplicación de sus normas en unas determinadas circunstancias; la jurisdicción judicial, que recoge los derechos y límites en la adjudicación de los casos a sus tribunales —en inglés, jurisdiction 
to adjudicate-, y la jurisdicción en aplicación de la ley, referida a los derechos y límites en la actuación de las fuerzas policiales y judiciales a la hora de conducir investigaciones y usar métodos coercitivos -en inglés, jurisdiction to enforce(Staker, 2014; Crawford, 2008, p. 456).

Tal y como indica O'Keefe (2016) en relación con el sofa que nos ocupa, la frase "sujeto a la jurisdicción exclusiva" no incluye la jurisdicción legislativa, sino únicamente la jurisdicción judicial y la jurisdicción de aplicación de la ley para el caso concreto del personal abarcado por el acuerdo, sin que ello suponga una renuncia a estos derechos de jurisdicción en sí mismos (p. 438). Concluye además, acertadamente este mismo autor, que la cesión que hacen los Estados parte vía artículo 12. 2. a del ER de su derecho consuetudinario a iniciar procedimientos penales por crímenes de genocidio, de guerra o lesa humanidad no se ve afectado por los sofa o cualquier otro acuerdo sobre inmunidades que ese Estado parte haya ratificado (p. 439).

También podría argumentarse que el fundamento de la jurisdicción de la CPI no se encuentra en la delegación que le hagan los Estados parte, ya que la jurisdicción sobre los crímenes internacionales procedería de la comunidad internacional. Según explica Carsten (2016),

La jurisdicción de la CPI no se deriva de la jurisdicción territorial o basada en la nacionalidad de un Estado específico, sino en un título más amplio de los Estados y de la comunidad internacional bajo el derecho internacional. Esta teoría establece que la justificación normativa para el castigo es independiente de la voluntad de los respectivos soberanos. Esto se justifica por el hecho de que los individuos se enfrentan a responsabilidad penal directa bajo el derecho internacional por crímenes internacionales. Los Estados ejercen esta jurisdicción en nombre de la comunidad internacional (p. 448). ${ }^{9}$

Dejando de lado los aciertos y desaciertos de los argumentos particulares expuestos, desde nuestro punto de vista debe hacerse una crítica general a la introducción del elemento de la cooperación estatal como factor clave que determine el interés de la justicia en la apertura de una investigación. Aunque es innegable que Estados Unidos no ha contraído obligaciones de cooperación con la CPI, la SCP parece olvidar que los Estados parte, entre ellos Afganistán, sí han asumido una serie de compromisos bajo la Parte IX del er. En su decisión en el asunto de Burundi, la 
SCP aclaró que los Estados parte no están obligados a cooperar en la etapa previa a la investigación, aunque la fiscalía y la CPI pueden solicitar su cooperación voluntaria (Sala de Cuestiones Preliminares, 2017, par. 15).

La falta de cooperación de Afganistán en la fase preliminar entra por tanto dentro de las expectativas habituales. Una vez que la investigación es autorizada, las obligaciones de cooperación del ER, incluyendo la obtención de pruebas y el arresto de sospechosos, devienen aplicables en su totalidad. El incumplimiento de estas obligaciones en el marco de una investigación ya autorizada supondría una vulneración del derecho internacional, con la posible intervención de la Asamblea de Estados parte y el CSNU, con unos costes políticos que, en muchas ocasiones, pueden alterar esa actitud inicial de no cooperación (Akande \& de Souza Días, 2019).

Tal y como hemos indicado, la falta de cooperación en la etapa preliminar por los Estados parte no supone un incumplimiento de las obligaciones asumidas en el ER. Desde nuestro punto de vista, valorar la falta de cooperación del Estado en esta etapa preliminar solo animaría a los Estados a no cooperar antes de la investigación, permitiéndoles defender sus intereses políticos sin necesidad de violar siquiera el ER, ya que la SCP se encargaría indirectamente de defender sus intereses al no autorizar la apertura de la investigación (Labuda, 2019).

\subsection{El uso eficiente de los recursos de la CPI}

El tercer elemento que tuvo en cuenta la SCP para valorar negativamente el interés de la justicia fue el riesgo de perder los ya de por sí escasos recursos con los que cuenta la CPI, que podrían emplearse en la investigación de asuntos más realistas o factibles. Este es, sin duda alguna, el elemento "más práctico" del que se sirvió la SCP en su polémica decisión (Kersten, 2019). Una vez más, la SCP no crea este elemento ex novo, podemos encontrar precedentes a favor de su uso a la hora de decidir la apertura de una investigación penal o el inicio de un enjuiciamiento.

Así, por ejemplo, Wirth (2003) resalta que Estados como Alemania introdujeron en la legislación que implementa el ER algunos principios que permiten ejercer cierta discrecionalidad en la investigación de los casos para prevenir el desperdicio de recursos en investigaciones con pocas perspectivas de éxito por encontrarse el sospechoso fuera de Alemania o ya inmerso en un procedimiento judicial en otro Estado (p. 160), algo que países como Australia también consideran en sus investigaciones puramente domésticas (Australia's Federal Prosecutor Service, s. d.). En su opinión parcialmente disidente en el asunto Jelisic ante el Tribunal Penal Internacional para la ex-Yugoslavia (TPIY), el juez Wald aceptó igualmente que "resources of the Tribunal are stretched thin and there may well be reason to prioritise cases involving 
allegations of State-planned and executed crimes, rather than individualistic or opportunistic crimes" (TPIY, 2001, par. 2).

De los distintos criterios utilizados por la SCP en su interpretación extensiva del concepto de "interés de la justicia", este es sin duda alguna el que más críticas merece. Tal y como indicó en su opinión el juez Wald,

Any such decision based on "judicial economy" inevitably reflects judges' views as to which cases are "worthy" and which are not. That, however, is the job of the Prosecutor who must calibrate legal and policy considerations in making her choices on how to utilise limited resources. To recognise a parallel power in judges to accept or reject cases on extra-legal grounds invites challenges to their impartiality as exclusively definers and interpreters of the law (TPIY, 2001, par. 14).

En el caso concreto de la CPI, el artículo 42.2 del ER establece que "el fiscal tendrá plena autoridad para dirigir y administrar la fiscalía, con inclusión del personal, las instalaciones y otros recursos". El objetivo de este precepto es asegurar la independencia de la fiscalía en el ejercicio de sus obligaciones estatutarias (Chinedu Ologbuo, 2018, p. 324), que ha sido utilizado por esta en el asunto de Afganistán para calificar el control realizado aquí por la SCP como de ultra vires (Oficina del Fiscal, 2019, par. 142). A ello suma, como bien se indica en el mismo escrito, que el control sobre los recursos corresponde a la Asamblea de Estados parte y no a los órganos judiciales, en virtud del artículo 112. 2. b del ER.

La introducción de consideraciones de eficiencia económica en el concepto del "interés de la justicia" a la hora de autorizar una investigación pone asimismo en peligro la propia legitimidad de la CPI. Mientras que la persecución de los crímenes más graves contra la humanidad es uno de los objetivos del derecho internacional penal, en general, y de la CPI, en particular, el criterio de la eficiencia económica, pese a ser mencionado en los ordenamientos internos, no parece ser, o al menos no debería ser, uno de los valores básicos a nivel internacional (Marston Danner, 2003, p. 545). Tal y como señala Webb (2005):

la cantidad de recursos temporales y financieros que serían empleados en un juicio debería ser un criterio a la hora de valorar el interés de la justicia, pero no debería ser un criterio decisivo. La eficiencia es un objetivo de gran valor, pero no es el propósito de la CPI (p. 342)..$^{10}$

10

Traducción propia. 


\section{La decisión de la SA: una aproximación no excluyente}

Los seguidores de la interpretación doctrinal restrictiva del concepto de "interés de la justicia" defienden, como hemos introducido, que únicamente sean valorados los elementos recogidos literalmente en el artículo 53. 1. c del ER: la gravedad de los crímenes y los intereses de las víctimas. Esta visión, no obstante, parece chocar con la redacción del precepto, el cual señala que la oficina del fiscal debe considerar si "aun teniendo en cuenta la gravedad del crimen y los intereses de las víctimas, una investigación no redundaría en interés de la justicia”. Una interpretación literal nos llevaría a concluir que la expresión "aun teniendo en cuenta” coloca la gravedad del crimen y los intereses de las víctimas como elementos independientes y de análisis previo al interés de la justicia.

En esta línea, el uso de la conjunción "nonetheless" en inglés ha llevado a la doctrina mayoritaria a defender que el artículo 53. 1. c del ER separa por un lado la gravedad de la justicia y los intereses de las víctimas del interés de la justicia, de manera que los primeros no deben tenerse en cuenta como elementos en la definición del segundo (Gallavin, 2003, p. 185). La gravedad de los crímenes y los intereses de las víctimas constituirían factores de contrapeso al "interés de la justicia” (Schabas, 2017, 236), de manera que la fiscalía "a un lado de la balanza ha de poner la gravedad de los crímenes y los intereses de las víctimas; al otro, el interés de la justicia" (Cárdenas Aravena, 2011, 26). Esta interpretación literal, sin duda convincente, parece haber quedado excluida tras la breve decisión al respecto de la sA en el asunto de Afganistán, en la que indica que estos dos son los dos elementos imprescindibles para realizar un adecuado análisis del interés de la justicia.

\subsection{La gravedad del crimen}

La gravedad del crimen es un elemento recurrente a lo largo del texto del ER. Introducido en el preámbulo a la hora de marcar el propósito de la CPI, lo encontramos de nuevo tanto en el test de admisibilidad del artículo 17. 1. d, como en el requisito del interés de la justicia del 53. 1. c del ER, lo que, como veremos, a dado pie a varios análisis doctrinales. Es indudable que, si el interés de la justicia es un concepto polémico por no estar definido en el ER, su definición se torna aún más problemática si se hace depender de otro concepto indefinido y multifacético como es el de la gravedad del crimen.

La pregunta básica que puede surgir es ¿tiene alguna relevancia la gravedad del crimen si esta ya ha sido analizada previamente bajo la óptica de la admisibilidad? O, en otras palabras, ¿se trata de un mero recordatorio de la importancia de este elemento? Algunos autores consideran que el hecho de que ya se haya superado 
la barrera del artículo 17. 1. d del ER hace surgir "dudas en torno a la influencia que pudiera llegar a ejercer la 'gravedad' del crimen en la valoración del interés de la justicia ya que, generalmente, todos merecerán el más severo reproche" (Loyo Cabezudo, 2021, 181), de manera que es el examen bajo dicho artículo el que realmente determina la selección o no de una situación para su potencial investigación (Clark, 2008, 37). Esto parece dar a entender la fiscalía en su Policy Paper sobre el interés de la justicia al señalar que, antes de proceder a analizar la gravedad del crimen en el marco del interés de la justicia, necesariamente tendrá que haber determinado ya si el crimen ha alcanzado la gravedad suficiente en el examen de admisibilidad (Oficina del Fiscal, 2007, p. 5).

Por el contrario, hay quien considera que esta segunda mención es una nueva oportunidad para la fiscalía de rechazar el caso, aun cuando supere el umbral de gravedad del artículo 17. 1. d por razones distintas como la existencia de otras situaciones más serias que requieran de una investigación con mayor urgencia (Bergsmo \& Kruger, 2008, 1071). Turone (2002) llega incluso a mencionar que se trata de una suerte de lex specialis, de manera que el análisis de la gravedad del crimen se produce únicamente bajo el paraguas del 53. 1. c, excluyendo cualquier otro precepto (p. 1153).

Si optamos por esta segunda visión, puede considerarse necesario determinar cuáles son las diferencias en el elemento de la "gravedad del crimen" en los distintos preceptos. Tras analizar la jurisprudencia de los tribunales ad hoc y de la propia CPI, autores como Ochi (2016) distinguen entre la gravedad del crimen en el marco del examen de un caso - teniendo aquí en cuenta únicamente la naturaleza de los propios crímenes y la perspectiva de las víctimas- y la gravedad de la situación - en cuyo caso se tendrían en cuenta además factores personales del acusado, como su papel en la comisión o su rango dentro de la organización- $-{ }^{11}$ No obstante, en el escenario de Afganistán, y dado que se trata de determinar la apertura o no de una investigación $-\mathrm{y}$ no de un caso concreto-, resulta de mayor interés la categorización realizada por otros autores según distintos niveles de gravedad, que recoge además la diferencia entre situaciones y procedimientos. Para McAuliffe de Guzman (2009), la gravedad juega dos papeles: uno estricto como base normativa para la determinación de la jurisdicción de la CPI y otro relativo como criterio que permite a la fiscalía una cierta discrecionalidad en la selección de casos y asuntos (pp. 1405-1406).

\footnotetext{
11 Mientras que el término "situación" se refiere a los límites dentro de los cuales la CPı determina si hay una base razonable para iniciar una investigación y los parámetros jurisdiccionales pertinentes (principalmente personales y territoriales), el término "caso" es más específico y se refiere a incidentes concretos durante los cuales uno o más crímenes dentro de la jurisdicción de la cPI han sido cometidos por uno o más sospechosos (Rastan, 2014).
} 
A partir de estos dos conceptos de gravedad, en lo que aquí nos interesa, Stegmiller (2014) distingue el criterio de "gravedad jurídica" en relación con la admisibilidad de la situación bajo el artículo 17. 1. d, y el criterio de "gravedad relativa" en lo que se refiere al interés de la justicia en la investigación de situaciones bajo el artículo 53. 1. c del ER. Por un lado, el requisito de la "gravedad jurídica" exigiría cumplir un umbral de exigencia relativamente bajo, basado en factores cuantitativos y objetivos que dejan poco margen de discrecionalidad a la fiscalía (Stegmiller, 2014, p. 619). Por otro lado, la "gravedad relativa" supone un umbral de exigencia más elevado que depende de factores no solo cuantitativos sino cualitativos, así como una comparación con otras situaciones investigadas, que permite una mayor discrecionalidad a la fiscalía a la hora de considerar la gravedad en el "interés de la justicia".

La decisión de la sA del 5 de marzo de 2020 en el asunto de Afganistán, más allá de establecer que la gravedad del crimen debe tenerse en cuenta a la hora de valorar el interés de la justicia, no aporta ningún dato sobre el umbral de gravedad que debe analizarse en este apartado. Si seguimos la visión ofrecida por la fiscalía en su Policy Paper - nos encontremos en la etapa de admisibilidad o en el contexto del artículo 53. 1. c-, los elementos a tener en cuenta según la regla 29 de la oficina de la fiscalía serían la escala, la naturaleza y el modo de comisión e impacto de los crímenes ("Regulations of the Office", 2009).

Un análisis de la SCP en esta última línea probablemente habría arrojado un resultado positivo. A la hora de considerar superado el umbral de gravedad en su análisis de admisibilidad del asunto de Afganistán, la SCP tuvo en cuenta de manera conjunta una serie de factores tanto cualitativos como cuantitativos - desviándose tanto de la doctrina que diferencia entre la gravedad en situaciones y casos, como de los factores cuantitativos en la admisibilidad y cualitativos en el interés de la justicia - entre los que se incluyen la naturaleza de crímenes como la tortura; la perspectiva de las víctimas y el papel ejercido por los sospechosos; el nivel de responsabilidad de los sospechosos; el elevado número de víctimas; el impacto en las víctimas, en especial las devastadoras consecuencias por la violencia recurrente sobre civiles inocentes, seleccionados como objetivos de forma recurrente, o la comisión a gran escala de los crímenes durante un periodo prolongado de tiempo (Sala de Cuestiones Preliminares II, 2019, pars. 80-86).

Por el contrario, la consideración del criterio de la "gravedad relativa" podría haber arrojado un resultado negativo, ya que permitiría tener en cuenta las necesidades prácticas y los recursos disponibles de la CPI para dedicarlos a los asuntos más graves (McAuliffe de Guzman, 2009, p. 1414; Stegmiller, 2014, p. 636). De hecho, como vimos con anterioridad, la SCP tuvo en cuenta este elemento práctico - si bien no 
dentro del análisis de la gravedad expresamente- en el marco del "interés de la justicia" a la hora de rechazar la apertura de la investigación.

\subsection{Los intereses de las víctimas}

La conexión entre los intereses de las víctimas y el interés de la justicia se encuentra de manera repetida en los escasos documentos oficiales en la materia. En su Policy Paper, la Fiscalía reconoce expresamente el papel vital que tienen los intereses de las víctimas en las distintas etapas del procedimiento ante la CPI (Oficinal de Fiscal, 2007, p. 5). Según este documento, la referencia en el artículo 53. 1. c a los intereses de las víctimas significa generalmente la apertura de una investigación. No obstante, no es este siempre el caso. Los intereses de las víctimas también pueden llevar al rechazo de la apertura de la investigación, ya que deben tenerse en cuenta todos los puntos de vista (Oficina del Fiscal, 2007, p. 5). Para determinar estos intereses, la fiscalía lleva a cabo diálogos no solo con las víctimas directas de los crímenes, sino también con otros intermediarios y representantes de las comunidades locales que puedan dar distintas perspectivas de la situación a investigar (Oficina del Fiscal, 2007, p. 7).

Un ejemplo de esta línea de actuación por parte de la fiscalía la encontramos en la situación de Georgia. Aunque la fiscalía comenzó indicando, tal y como hace la sA, que no le corresponde justificar el interés de la justicia cuando solicita la apertura de una investigación, dedica a ello un breve análisis en el que destaca la importancia de los intereses de las víctimas. La solicitud de apertura de la investigación incluye, dentro del apartado del interés de la justicia, una sección en la que informa sobre las reuniones con los georgianos que alegan haber sido víctimas de crímenes bajo la jurisdicción de la CPI, declaraciones de testigos, observaciones de organizaciones de derechos humanos y miembros de la administración pública de Georgia, señalando que, de las declaraciones de las víctimas y de los intermediarios y representantes, no se desprende que la apertura de una investigación sería contraria al interés de la justicia (Oficinal del Fiscal, 2015, pars. 340-343).

Los peligros derivados del precedente que podría haber asentado la SCP en el asunto de Afganistán probablemente han motivado que, más recientemente, la SCP haya vinculado los intereses de las víctimas con el interés de la justicia. En su decisión de noviembre de 2019, en la que autorizó la apertura de la investigación en la situación de Myanmar, la SCP dedicó una sección completa al interés de la justicia y, dentro de ella, a los intereses de las víctimas. En particular, resaltó las declaraciones según las cuales el círculo de violencia contra los rohingyas solo puede conseguirse a través de la justicia y la exigencia de responsabilidad, así como del 
compromiso y deseo de las víctimas de participar en los procesos ante la CPI con el objetivo de llevar a los presuntos responsables ante la justicia (Sala de Cuestiones Preliminares II, 2019, par. 38).

La consideración de los intereses de las víctimas en el análisis del interés de la justicia, fuertemente defendida por parte de la doctrina y de la sociedad civil, es apoyada por la sA en el asunto de Afganistán y por la SCP en Myanmar. Sus razonamientos, basados en informes proporcionados por la fiscalía sobre casos específicos y las declaraciones de los representantes legales de las víctimas, contrasta con la manifiesta falta de información que tenía en sus manos la SCP cuando afirmó que la apertura de una investigación en Afganistán iría en contra de los intereses de las víctimas, creándoles falsas expectativas de obtener justicia, que probablemente ocasionaría hostilidad hacia la labor de la CPI (Sala de Cuestiones Preliminares II, 2019, par. 96).

\section{Conclusiones}

La introducción de elementos temporales y económicos en la valoración del requisito del "interés de la justicia" por la SCP en el asunto de Afganistán casi conduce a la CPI al borde de una profunda crisis de identidad y credibilidad. Tal y como señala Orihuela (2019), si la credibilidad de la CPI ya había sido atacada con anterioridad y su futuro era incierto, la decisión de la scP en el asunto de Afganistán pudo haber causado su propia destrucción (p. 30). Hasta el momento, los riesgos en el uso del interés de la justicia se asociaban a la discrecionalidad que puede otorgar a la fiscalía, a la hora de decidir sobre la solicitud o no de apertura de una investigación, aunque, por ahora, esta ha realizado una interpretación restrictiva al respecto. De manera inesperada, la inclusión de estos elementos ha venido del órgano que tiene atribuido el control jurisdiccional de las actuaciones de la fiscalía: la SCP. El hecho de que esta sala decidiera, por primera vez en dos décadas, entrar a analizar este requisito para rechazar la apertura de un asunto tan polémico por incluir la investigación de personal estadounidense constituye una dura losa sobre la legitimidad de este joven tribunal internacional.

En una aplaudida decisión, la SA salvó la situación estableciendo que la SCP había cometido un error de derecho a la hora de entrar a analizar el requisito del interés de la justicia, algo posible únicamente cuando la fiscalía ha decidido no abrir una investigación por este motivo. No obstante, y esta ha sido la principal motivación de nuestro estudio, no realiza un análisis sustantivo del interés de la justicia ni descarta de forma expresa los elementos utilizados por la SCP, más allá de criticar que el análisis no contaba con suficiente información que respaldase la decisión 
final. Por lo tanto, resulta de vital importancia conocer los elementos que pueden influir en la formación del requisito del "interés de la justicia" para la apertura de investigaciones en un futuro. La sa solo menciona la gravedad de los crímenes y los intereses de las víctimas, para resolver, en principio, el debate doctrinal sobre si estos elementos formaban parte integrante del interés de la justicia o si, por el contrario, debían valorarse en contraposición por la interpretación literal del artículo 53. 1. c del ER.

En cuanto a los tres elementos específicamente mencionados por la SCP en su interpretación amplia del "interés de la justicia", los relativos al uso eficiente de los recursos económicos y al lapso entre la investigación deberían ser, bajo nuestro punto de vista, completamente descartados. El primero de ellos, porque corresponde a una cuestión de organización propia y autónoma de la fiscalía, y el segundo, porque implicaría la impunidad de los crímenes por el paso del tiempo, aun cuando existen sobrados ejemplos que muestran lo contrario. En cuanto al tercer elemento relativo a la falta de cooperación de los Estados, aceptamos que, desde la perspectiva del derecho internacional, no puede obligarse a cooperar a Estados que no son parte del ER. No obstante, extender en la etapa preliminar este razonamiento a los que sí lo han ratificado haría perder la razón de ser de las obligaciones que se activan en la fase de la investigación, animando a los Estados parte a no cooperar según sus intereses, sin que ello suponga siquiera una ruptura de sus obligaciones convencionales.

Como señaló Kelsen (1991), no es posible responder de forma definitiva y absoluta a la pregunta de qué es la justicia. Ni siquiera cuando el interrogante surge dentro de una misma organización internacional, ya que puede depender del órgano que lo plantea, las competencias que tiene atribuidas o incluso la propia información de la que se dispone en el asunto concreto. No hemos pretendido aquí, por tanto, ofrecer más que un análisis de los elementos que pueden equilibrar en uno u otro sentido el concepto de "interés de la justicia". De este modo, el análisis realizado es prueba de que la complejidad de los asuntos a los que se enfrenta la justicia penal internacional impide que nos pronunciemos a favor de una interpretación restrictiva o extensiva en términos absolutos. Será necesario esperar a desarrollos futuros para ver si la sA realiza un análisis más exhaustivo sobre los elementos que pueden estar presentes en el concepto del "interés de la justicia". Lo único que podemos concluir con certeza es que los valores recogidos en el ER son los que deben orientar esta definición, el primero de ellos es evitar la impunidad "de los crímenes más graves de trascendencia para la comunidad internacional en su conjunto". 


\section{Referencias}

\section{Doctrina}

Akande, D. (2003). The jurisdiction of the International Criminal Court, over nationals of non-parties: Legal basis and limits. Journal of International Criminal Justice, 1(3), 618-650. https://doi.org/10.1093/jicj/1.3.618

Ambos, K. (2016). Treatise on international criminal law, Vol. III. Oxford University Press.

Bantekas, I. (2010). International criminal law. Hart Publishing.

Bantekas, I. (2011). Criminal jurisdiction of states under international law. Max Planck Encyclopedia of Public International Law.

Bergsmo, M., \& Kruger, P (2008). Investigation and prosecution. En O. Triffterer (Ed.), Commentary on the Rome Statute of the International Criminal Court (pp. 1065-1076). Hart Publishing.

Bosco, D. (2020). Putting the prosecutor on a clock? Responding to variance in the length of preliminary examinations. AJIL Unbound, 112 (Symposium on the Rome Statute at Twenty), 158-162. https://doi.org/10.1017/aju.2018.53

Cárdenas Aravena, C. M. (2011). Revisión crítica del criterio «interés de la justicia» como razón para no abrir una investigación o no iniciar un enjuiciamiento ante la Corte Penal Internacional. Revista de Derecho, 1, 21-47.

Carsten, S. (2016). The ICC, pre-existing jurisdictional treaty regimes, and the limits of the nemo dat quod non habet doctrine -A reply to Michael Newton. Vanderbilt Journal of Transnational Law, 49(2), 443-454.

Clark, P. (2008). Law, politics and pragmatism: The ICC and case selection in the Democratic Republic of Congo and Uganda. En N. Waddell \& P. Clark (Eds.), Courting conflict? Justice, peace and the ICC in Africa (pp. 37-45). Royal African Society.

Crawford, J. (2008). Brownlie's principles of public international law. Oxford University Press.

Dancy, G., \& Montal, F. (2017). Unintended positive complementarity: Why International Criminal Court investigations may increase domestic human rights prosecutions. American Journal of International Law, 111(3), 689-723. https://doi.org/10.1017/ajil.2017.70 
Danilenko, G. M. (2000). The statute of the International Criminal Court and third states. Michigan Journal of International Law, 21(3), 445-494. https://repository.law.umich.edu/mjil/vol21/iss3/3

Dannenbaum, T. (2020). Legitimacy in war and punishment: The Security Council and the ICC. En K. J. Heller et al. (Eds.), The Oxford handbook of international criminal law (pp. 129-154). Oxford University Press.

De Souza Dias, T. (2017). Interests of justice: Defining the scope of prosecutorial discretion in article 53(1)(c) and (2)(c) of the Rome Statute of the International Criminal Court. Leiden Journal of International Law, 2017, 731-751.

Dörr, O., \& Schmalenbach, K. (2018). Article 34: general rule regarding third States. En Autores, Vienna Convention on the law of treaties: A commentary (pp. 613-615). Springer.

Gómez Colomer, J. L. (2002). La investigación del crimen en el proceso penal ante la Corte Penal Internacional. En J. Cardona Llorens et al. (Coords.), La Corte Penal Internacional: un estudio interdisciplinar (pp. 227-324). Tirant lo Blanch.

Gallavin, C. (2003). Article 53 of the Rome Statute of the International Criminal Court: In the interests of justice? King's Law Journal, 4, 179-198.

Kelsen, H. (1991). ¿Qué es la justicia? (Edición española a cargo de Albert Calsamiglia). Editorial Ariel.

Loyo Cabezudo, J. (2021). La llave del «interés de la justicia» en el Estatuto de Roma: su controvertido empleo en el caso de Afganistán. Revista Española de Derecho Internacional, 73(1), 165-186.

Magnoux, C. (2017). The sound of silence: Le pouvoir discrétionnaire du procureur de la Cour pénale internationale à travers l'utilisation des critères d'intérêts de la justice et de gravité lors de louverture d'une enquête. Revue Québécoise de Droit International, 9-36.

Marston Danner, A. (2003). Enhancing the legitimacy and accountability of prosecutorial discretion at the International Criminal Court. American Journal of International Law, 97, 510-552.

McAuliffe de Guzman, M. (2008). Gravity and the legitimacy of the International Criminal Court. Fordham Journal of International Law, 32(5), 1400-1465.

Newton, M. A. (2016). How the International Criminal Court threatens treaty norms. Vanderbilt Journal of Transnational Law, 49(2), 371-442. 
Ochi, M. (2016). Gravity threshold before the International Criminal Court: An overview of the court's practice. ICD Brief.

Olásolo Alonso, H. (2005). The triggering procedure of the International Criminal Court, procedural treatment of the principle of complementarity, and the role of office of the prosecutor. International Criminal Law Review, 5, 121-146.

Olásolo Alonso, H. (2012). El principio de complementariedad y las estrategias de actuación de la Corte Penal Internacional en la fase de examen preliminar: ¿Por qué la Corte Penal Internacional mantiene su examen preliminar, pero no abre una investigación, sobre la situación en Colombia? Revista Electrónica de Estudios Internacionales, 24, 1-45.

O'Keefe, R. (2016). Quid not quantum: A comment on how the International Criminal Court threatens treaty norms. Vanderbilt Journal of Transnational Law, 49(2), 433-442.

Orihuela Calatayud, E. (2019). When are there substantial reasons to believe that an investigation of core crimes would not serve the interests of justice? Spanish Yearbook of International Law, 23, 9-30.

Orihuela Calatayud, E. (2020). La autorización para investigar los crímenes cometidos en Afganistán. Luces y sombras de la sentencia, de 5 de marzo de 2020, de la Sala de Apelaciones de la Corte Penal Internacional. Revista Electrónica de Estudios Internacionales, 39, 1-19.

Poltronieri Rossetti, L. (2019). The pre-trial chamber's Afghanistan decision: A step too far in the judicial review of prosecutorial discretion? Journal of International Criminal Justice, 17, 585-608.

Pues, A. (2017). Towards the 'golden hour'? A critical exploration of the length of preliminary examinations. Journal of International Criminal Justice, 15, 435-453.

Rastan, R. (2014). Situation and case: defining the parameters. En C. Stahn \& M. M. El Zeidy (Eds.), The International Criminal Court and complementarity: From theory to practice (pp. 421-459). Cambridge University Press.

Ryngaert, C. (2015). Jurisdiction in international law. Oxford University Press.

Schabas, W. (2017). An introduction to the International Criminal Court. Cambridge University Press. 
Seils, P. (2015). Putting complementarity in its place. En C. Stahn (Ed.), The law and practice of the International Criminal Court (pp. 305-327). Oxford University Press.

Staker, C. (2014). Jurisdiction. In D. Evans (Ed.), International law (pp. 309-333). Oxford University Press.

Stegmiller, I. (2014). Interpretative gravity under the Rome Statute: Identifying common gravity criteria. En C. Stahn \& M. M. El Zeidy (Eds.), The International Criminal Court and complementarity: From theory to practice (pp. 603-641). Cambridge University Press.

Turone, G. (2002). Powers and duties of the prosecutor. En A. Cassese, P. Gaeta, \& J. Jones (Eds.), The Rome Statute of the ICC: A commentary (pp. 1138-1180). Oxford University Press.

Vagias, M. (2014). The territorial jurisdiction of the International Criminal Court. Cambridge University Press.

Vierdag, E. W. (1982). The law governing treaty relations between parties to the Vienna Convention on the law of treaties and states not party to the convention. American Journal of International Law, 76(4), 779-801.

Webb, P. (2005). The ICC prosecutor's discretion not to proceed in the "interests of justice. Criminal Law Quarterly, 50, 305-348.

Wenqi, Z. (2006). On co-operation by states not party to the International Criminal Court. International Review of the Red Cross, 88(861), 87-110.

Wirth, S. (2003). Germany's new international crimes code: Bringing a case to court. Journal of International Criminal Justice, 1(1), 151-168.

\section{Jurisprudencia}

CPI, Sala de Apelaciones. (30 de noviembre de 2006). Decision requesting information on the status of the preliminary examination of the situation in the Central African Republic, ICC-01/05.

CPI, Pre-Trail Chamber. (25 de octubre de 2017). Decision pursuant to Article 15 of the Rome Statute on the authorization of an investigation into the situation in the Republic of Burundi, ICC-01/17-X. 
CPI, Pre-Trial Chamber II. (12 de abril de 2019). Decision pursuant to Article 15 of the Rome Statute on the authorisation of an investigation into the situation in the Islamic Republic of Afghanistan, ICC-02/17.

CPI, Sala de Apelaciones. (5 de marzo de 2020). Judgment on the appeal against the decision on the authorisation of an investigation into the situation in the Islamic Republic of Afghanistan, ICC-02/17 OA4.

CPJI. (7 de septiembre de 1927). The case of the S. S. Lotus (France v. Turkey).

TPIY. (5 de julio de 2001). Prosecutor v. Goran Jelisi, partly dissenting opinion of judge Wald, IT-95-10-A.

United States v. Hastings, 847 F. 2d 920,923 (1st Cir., 1998).

Schooner Exchange v. McFaddon, 11 U.S. (7 Granch) 116, 136, 3 L.Ed. 287 (1812).

\section{Otras fuentes}

Akande, D., \& de Souza Días, T. (2019). The ICC pre-trial chamber decision on the situation in Afghanistan: A few thoughts on the interests of justice. EJIL: Talk! https://www.ejiltalk.org/the-icc-pre-trial-chamber-decision-on-thesituation-in-afghanistan-a-few-thoughts-on-the-interests-of-justice/

Australia's Federal Prosecution Service. (s. f.). Guidelines for the making of decisions in the prosecution process. https://www.cdpp.gov.au/sites/default/files/Prosecution-Policy-of-the-Commonwealth_0.pdf

CPI. Appeals Hearing. (5 de diciembre de 2019). ICC-02/17-T-002. https://www. icc-cpi.int/Transcripts/CR2019_07401.PDF

CPI, Oficina del Fiscal. (2007). Policy paper on the interests of justice. https://www. icc-cpi.int/nr/rdonlyres/772c95c9-f54d-4321-bf09-73422bb23528/143640/ iccotpinterestsofjustice.pdf

CPI, Oficina del Fiscal. (2015). Strategic Plan 2016 - 2018. https://www.icc-cpi.int/ iccdocs/otp/en-otp_strategic_plan_2016-2018.pdf

Legal representatives of the victims, Victims' Appeal Brief. (24 de junio de 2019). ICC-02/17 OA.

CPI, Oficina del Fiscal. (7 de junio de 2019). Request for leave to appeal the "Decision pursuant to Article 15 of the Rome Statute on the authorisation of an investigation into the situation in the Islamic Republic of Afghanistan", ICC-02/17. 
Kersten, M. (2019). Whither the aspirational ICC, welcome the 'practical' court? EJIL Talk. https://www.ejiltalk.org/whither-the-aspirational-icc-welcome-the-practical-court/

Labuda, P. I. (2019). A neo-colonial court for weak states? Not quite. Making sense of the International Criminal Court's Afghanistan decision. EJIL: Talk! https://www.ejiltalk.org/a-neo-colonial-court-for-weak-states-not-quitemaking-sense-of-the-international-criminal-courts-afghanistan-decision/

"United States Imposes Economic Sanctions and Visa Restrictions on International Criminal Court Officials", American Journal of International Law, 115(1), 138-140.

Vasilev, S. (2019). Not just another 'crisis': Could the blocking of the Afghanistan investigation spell the end of the ICC? (Part I). EJIL: Talk! https://www.ejiltalk. org/not-just-another-crisis-could-the-blocking-of-the-afghanistan investigation-spell-the-end-of-the-icc-part-ii/ 